\title{
Grundlagen und Anwendungen einer neuen Diffusionsmethode in flüssiger Phase 1
}

Teil I: Die Steigerung des Diffusionstransportes durch Pulsationsdiffusion

\author{
G. Dreyer, E. Kahrig, D. Kirstein, J. Erpenbeck und Fr. Lange
}

Institut für Biophysik der Deutschen Akademie der Wissenschaften zu Berlin

(Direktor: Prof. Dr. habil. Kн. Loнs)

(Z. Naturforsch. 23 a, 498-503 [1968]; eingegangen am 18. Dezember 1967)

\begin{abstract}
Es wird eine neue Diffusionsmethode angegeben, die darauf beruht, daß sich bei pulsierender Flüssigkeitsströmung in einem porösen oder lamellierten Diaphragma an den Poren- bzw. Lamellenwänden sehr stabile Flüssigkeitsschichten ausbilden, die, in wechselnder Richtung von sehr unterschiedlich konzentrierten Lösungen bespült, hohe Konzentrationsgradienten bei großen Austauschflächen zulassen. Bei geeigneter Anordnung kann der Stofftransport um mehrere Zehnerpotenzen gegenüber den bekannten Anordnungen gesteigert werden bei gleichzeitig sehr erheblich verminderten konvektiven Störungen.
\end{abstract}

Die vorliegende Arbeit entstand aus dem Bedürfnis, ein von Lange und Mitarbeitern in der Sowjetunion entwickeltes Zirkulations-Diffusions-Trennverfahren für Gasgemische ${ }^{4-6}$ in die flüssige Phase zu übertragen.

Voraussetzung dafür war aber, die von der Langsamkeit des Diffusionsprozesses in Flüssigkeiten herrührenden Schwierigkeiten zu überwinden. Trotz entsprechend größerer Dichte zwingen die gegenüber der Gasphase etwa vier bis fünf Zehnerpotenzen kleineren Diffusionskoeffizienten in Flüssigkeiten zu außerordentlich kurzen Diffusionswegen bei großem Diffusionsquerschnitt, will man einen mit der Gasphase vergleichbaren Diffusionstransport erreichen.

In der Regel bestehen Diffusionsanordnungen aus zwei miteinander verbundenen Vorratsgefäßen, die mit unterschiedlich konzentrierten Lösungen gefüllt sind. Der Diffusionsaustausch erfolgt durch ein Verbindungselement, das zur Vermeidung von Durchmischungsvorgängen zwischen beiden Vorratsgefäßen eng lamelliert ist oder aus einem porösen gesinterten Material besteht. Eine Verkürzung des Diffusionsweges bei großem Diffusionsquerschnitt ist dabei durch das Auftreten konvektiver Störungen des Diffusionsprozesses begrenzt.

Die genannten Schwierigkeiten treten auch bei der Bestimmung von Diffusionskoeffizienten im stationären oder quasistationären Zustand auf, so daß ein Prinzip zur Steigerung des Diffusionstransportes

1 Erste ausführliche Mitteilung der Ergebnisse einer Dissertationsschrift ${ }^{2,3}$, die unter der Leitung von $F_{R}$. LANGE durchgeführt wurde.

2 Fr. Lange u. G. Dreyer, DWP Nr. 54339 (Kl. 12 e, 3/04).

3 G. Dreyer, Inaugural-Dissertation, TH-Ilmenau 1967. auch für die Bestimmung von Diffusionskoeffizienten wünschenswert sein kann. Als geeignetes Prinzip erwies sich die Diffusion in pulsierender Flüssigkeitsströmung ${ }^{2,3}$.

\section{Grundlagen}

Es sei die Lösung eines Stoffes im Raum 1 von seinem reinen Lösungsmittel in der Kapillare (Lamelle) 2 und Raum 3 überschichtet. In einer konventionellen Anordnung wäre der Diffusionsweg durch die Länge

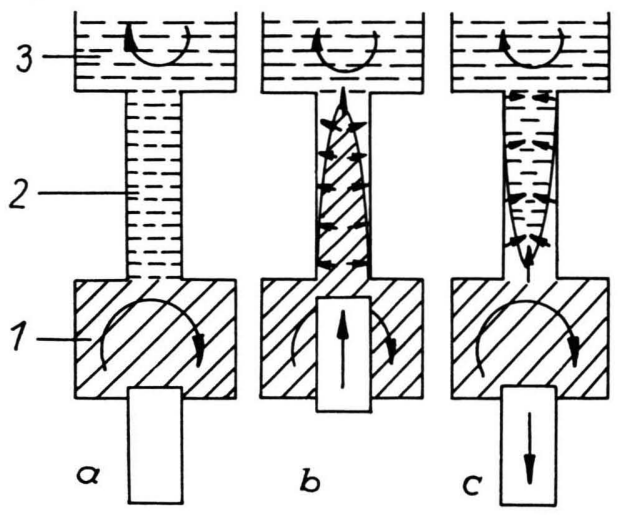

Abb. 1. Schematische Darstellung der Diffusion in pulsierender Flüssigkeitsströmung. a) Anordnung aus zwei Vorratsgefäßen 1 und 3, und einer Kapillare oder Lamelle 2. b) Aufwärts bewegter Stempel: Ausbildung des Lösungsparaboloids und Diffusion in Pfeilrichtung in die Randschicht. c) Abwärts bewegter Stempel: Ausbildung des Lösungsmittelparaboloids und Diffusion in Pfeilrichtung aus der Randschicht.

4 Fr. Lange, „Otsched o Rabote po Rasdelenie Isotopow“, Forschungsbericht Moskau 1947 (unveröffentlicht).

5 A. S. Kompanejez, Ann. Phys. 7. Folge, Bd. 8 (Heft 5-6) 303 [1961]

${ }^{6}$ Fr. Lange u. E. Kahrig, DWP Nr. 28316 (Kl. 12 e, 3/04). 
der Kapillare (Lamelle) und die Austauschfläche durch ihren Querschnitt gegeben. Bei pulsierender Flüssigkeitsströmung jedoch wird die Lösung aus Raum 1 beispielsweise durch einen Stempel in das Verbindungselement gedrückt, und es bildet sich ein Lösungsparaboloid (Hagen-Poiseuillesches Gesetz) aus, Abb. 1b. Die Diffusion erfolgt in Richtung der gezeichneten Pfeile senkrecht zur Paraboloidoberfläche. Damit reichert sich das das Paraboloid umgebende Lösungsmittel mit diffundierenden Teilchen an. Es soll als Zwischenmedium bezeichnet werden. Bei abwärts bewegtem Stempel wird das Lösungsparaboloid zurückgezogen, und es bildet sich als Folge ein Lösungsmittelparaboloid in umgekehrter Richtung aus, Abb. $1 \mathrm{c}$, in das die Diffusion nun aus dem Zwischenmedium erfolgt.

Bei erneut aufwärts bewegtem Stempel wiederholen sich die geschilderten Vorgänge, und die in das Lösungsmittelparaboloid diffundierten Teilchen gelangen in das obere Vorratsgefäß. Durch den periodischen Wechsel der Paraboloide und der Durchmischung in den Vorratsgefäßen können beträchtliche Konzentrationsgradienten aufrechterhalten werden, denn der Diffusionsweg ist nur von der Größenordnung des Kapillardurchmessers (Lamellenabstandes), und die Konzentrationsunterschiede zwischen den Paraboloiden bleiben stets von der Größe der Konzentrationsdifferenz der Vorratsgefäße. Die Austauschfläche ist durch die Oberfläche der Paraboloide gegenüber dem Querschnitt der Kapillare (Lamelle) außerordentlich vergrößert. Man wird ein Verbindungselement mit möglichst engen und langen Kapillaren oder Lamellen in dichter Packung anstreben.

Damit der Stofftransport nur durch Diffusion gegeben ist, darf das pulsierende Flüssigkeitsvolumen nur so groß sein, daß die Paraboloide nicht in das jeweils gegenüberliegende Vorratsgefäß eintreten können. Die pulsierende Flüssigkeitsströmung selbst hat keinen direkten Einfluß auf die Größe des Stofftransportes.

Im folgenden sei eine Abschätzung der geschilderten Pulsationsvorgänge in einer Kapillare gegeben:

In eindimensionaler Betrachtungsweise ist im Falle stationärer Diffusion der Diffusionstransport $J$ durch das 1. Ficksche Gesetz gegeben:

$$
J=-D \frac{\Delta c}{\Delta x} F
$$

( $D$ Diffusionskoeffizient; $\Delta c=c^{0}-c_{\mathrm{u}}$ Konzentrationsdifferenz zwischen der Konzentration im oberen Vorratsgefä $\beta c^{0}$ und der Konzentration im unteren Vorratsgefä $\beta c_{\mathrm{u}} ; \Delta x$ Diffusionsweg; $F$ Diffusionsquerschnitt).

Durch die Pulsation werden sowohl der Diffusionsweg $\Delta x$ als auch der Diffusionsquerschnitt $F$ beeinflußt.
Der Diffusionsquerschnitt ist durch die Paraboloidoberfläche gegeben,

$$
F=\frac{\pi r}{6 h^{2}}\left[\left(4 h^{2}+r^{2}\right)^{3 / 2}-r^{3}\right],
$$

wobei die Höhe des Paraboloids durch die periodische Stempelbewegung eine Funktion der Zeit ist:

$$
h(t)=\frac{H}{2}(1-\cos \omega t)
$$

( $H$ maximale Paraboloidhöhe bzw. Länge der Kapillare).

Das zeitliche Mittel von $h$ ist $\frac{1}{2} H$.

Die mittlere Paraboloidoberfläche bei Pulsation ist dann

$$
F=\frac{2 \pi r}{3 H^{2}}\left[\left(H^{2}+r^{2}\right)^{3 / 2}-r^{3}\right] .
$$

Es ist vom Prinzip der Pulsation her einleuchtend, daß man eine Anordnung mit $H \gg r$ anstreben wird. Der Ausdruck für den Diffusionsquerschnitt vereinfacht sich dann zu :

$$
F=\frac{2}{3} \pi r H .
$$

Zur Abschätzung des Diffusionsweges seien folgende Überlegungen vorausgeschickt:

Abb. 2 a zeigt das Lösungsparaboloid der Konzentration $c_{0}$ in einer Kapillare mit dem Radius $r$ und der Höhe $H$ und zwei Linien, die Punkte gleicher Konzentration im Zwischenmedium miteinander verbinden, beispielsweise von $c_{0} / 2$ und $c_{0} / 4$.

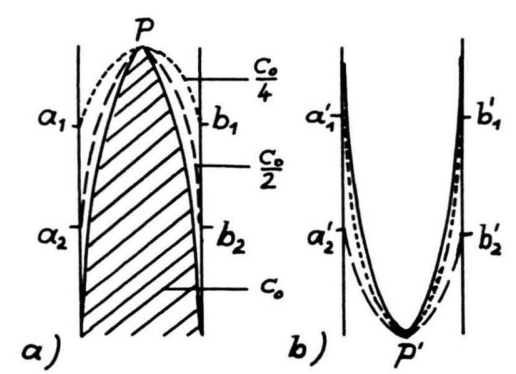

Abb. 2. Abschätzung des Diffusionsweges [Erläuterungen im Text].

Abb. 2 b zeigt die Phase der Pulsation, in der das Lösungsparaboloid wieder zurückgezogen ist und sich als Folge ein Lösungsmittelparaboloid der Konzentration $c=0$ ausgebildet hat. Damit gehen die Koordinaten des Punktes $\mathrm{P}$ in die des Punktes $\mathrm{P}^{\prime}$ über, während die Randpunkte unverändert bleiben (Strömungsgeschwindigkeit in der Randschicht gleich Null). Es zeigt sich damit, daß die Gebiete des Zwi- 
schenmediums, die dem Lösungsparaboloid am fernsten sind, im weiteren Verlauf der Pulsation dem Lösungsmittelparaboloid am nächsten sind; oder in anderen Worten: Der Konzentrationsgradient ändert im Verlauf der Pulsation seine Richtung in der Weise, daß die diffundierenden Teilchen zunächst bis in die äußerste Randschicht der Kapillare diffundieren müssen und aus dieser Randschicht wieder in Richtung auf die Kapillarachse.

Der Diffusionsweg ist damit angenähert gleich der doppelten mittleren Entfernung von der Oberfläche eines Paraboloids zur Kapillarwand. Diese Annahme setzt voraus, daß sich innerhalb des Lösungsparaboloids praktisch kein Konzentrationsgradient durch die herausdiffundierenden Teilchen ausbildet; eine Bedingung, die durch genügend häufigen Wechsel zwischen Ein- und Ausströmen der Lösung in der Kapillare und anschließender Durchmischung in den Vorratsgefäßen leicht zu realisieren ist.

In der in Abb. 3 dargestellten Form geben die Ordinaten

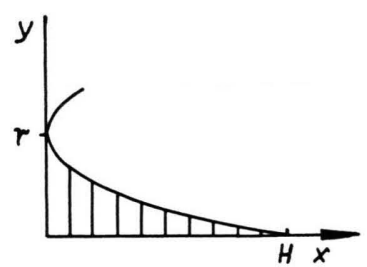

Abb. 3. Abschätzung des Diffusionsweges [Erläuterungen im Text].

$$
y=r(1-\sqrt{x / H})
$$

die Abstände vom Paraboloid zur Kapillarwand wieder. Die doppelte mittlere Entfernung von der Paraboloidoberfläche zur Kapillarwand ist dann:

$$
\begin{aligned}
& \Delta x \approx 2 \overline{y(x)}=\frac{2}{H} \int_{0}^{H} y(x) \mathrm{d} x, \\
& \Delta x \approx \frac{2}{3} r .
\end{aligned}
$$

Gehen wir mit den gefundenen Ausdrücken für den Diffusionsquerschnitt $F$ und dem Diffusionsweg $\Delta x$ in (1) ein und berücksichtigen wir die durchgeführte Näherung durch eine noch unbestimmte Konstante $K$, so erhalten wir für den Diffusionstransport bei Pulsationsdiffusion im stationären Fall ${ }^{3}$

$$
J=-K D \Delta c \pi H .
$$

Es ergibt sich der zunächst überraschende Sachverhalt, daß der Diffusionsstrom zwar abhängig von der Länge der Kapillare $H$, aber unabhängig von ihrem Durchmesser ist, d. h. eine Kapillare mit großem Durchmesser ist durch eine gleichlange engere Kapillare ersetzbar, ohne daß eine Verminderung des Stoffdurchsatzes eintritt. Dies bedeutet aber, daß man konvektive Störungen des Diffusionsprozesses ohne Verlust an Diffusionstransport in immer stärkerem Maße einschränken kann. Der in konventionellen Anordnungen begrenzende Kompromiß zwischen Diffusionstransport und Strömungswiderstand kann damit in einer Pulsationsdiffusionsanordnung überwunden werden.

\section{Ergebnisse}

Die experimentelle Prüfung der angegebenen Überlegungen muß also folgende Aussagen liefern:

1. Nachweis reiner Diffusionsvorgänge bei Pulsation;

2. Unabhängigkeit des Diffusionstransportes von der Pulsationsfrequenz bei genügend häufigem Wechsel der Paraboloide;

3. Unabhängigkeit des Diffusionstransportes vom Durchmesser der Kapillare;

4. Nachweis der linearen Abhängigkeit des Diffusionstransportes von der Kapillarlänge.

Abb. 4 zeigt die dazu aufgebaute Glasapparatur. Sie besteht im wesentlichen aus zwei thermostatierbaren Vorratsgefäßen, zwischen denen mittels Schliffverbindungen Kapillaren unterschiedlicher Länge bzw. unterschiedlichen Durchmessers auswechselbar eingefügt werden können. In diesen Kapillaren werden die geschilderten Pulsationsvorgänge erzeugt. Das untere Vorratsgefäß steht mit einem kleinen Gefäß in Verbindung, das durch eine Membran zur Erzeugung der Pulsationsvorgänge und einem seitlich angeschmolzenen Schliffhahn abgeschlossen ist. Auf das obere Vorratsgefäß ist eine ebenfalls auswechselbare Kapillare aufgesetzt, die bei gefüllter Apparatur durch die steigende und fallende Flüssigkeitssäule eine laufende Kontrolle des eingestellten Membranhubes erlaubt. Die versetzte Anordnung der Kapillaren beider Vorratsgefäße bewirkt bei schwingender Membran eine Durchmischung der in den Vorratsgefäßen enthaltenen Flüssigkeiten, so daß die Diffusionsvorgänge auf die Verbindungskapillare beschränkt bleiben.

Die Apparatur wird über den Schliffhahn bis zum oberen Rand der Verbindungskapillare mit der Lösung gefüllt, deren Diffusionstransport bestimmt werden soll, und das obere Vorratsgefäß einschließlich der Aufsatzkapillare mit Lösungsmittel. Dabei befindet sich die Membran an ihrem oberen Umkehrpunkt, so daß die Pulsationsvorgänge mit dem Lösungsmittelparaboloid beginnen. 


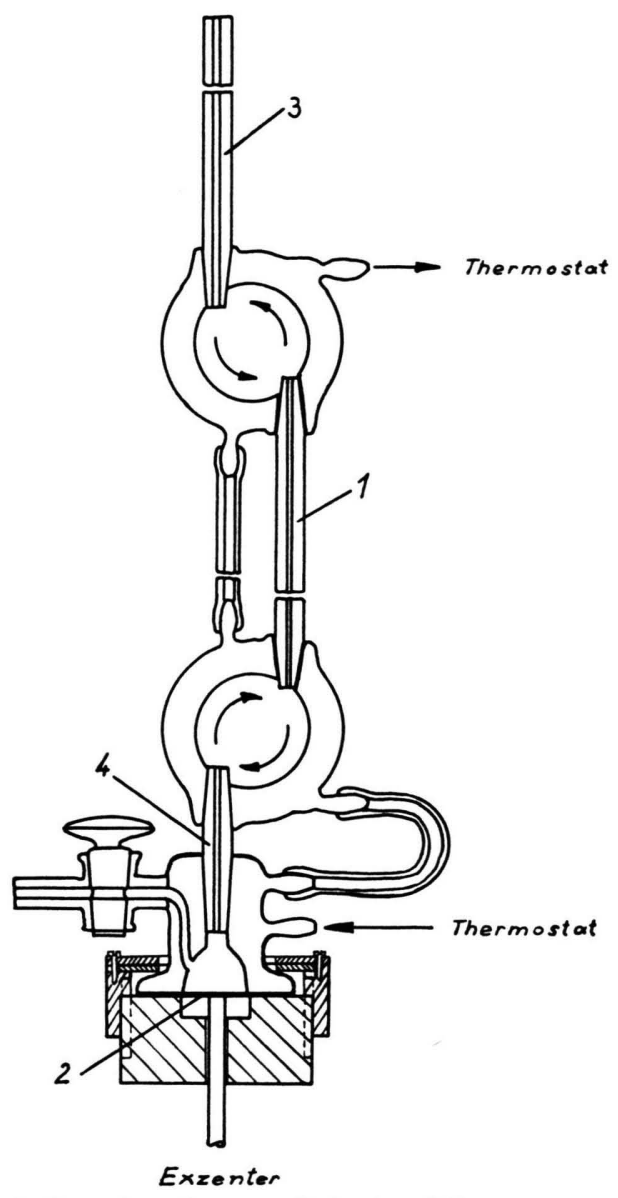

Abb. 4. Versuchsaufbau zur Pulsationsdiffusion in einer Kapillare 1 zwischen zwei thermostatierbaren Vorratsgefäßen. Die Huberzeugung erfolgt durch eine Membran 2, die Kontrolle des eingestellten Hubes an der Aufsatzkapillare 3 und die Durchmischung der Vorratsgefäße durch die versetzte Anordnung der Kapillaren 1, 3 und 4. Alle Kapillaren sind über Schliffverbindungen auswechselbar.

Die Konzentration im oberen Vorratsgefäß wächst zunächst relativ rasch, um nach einer gewissen Zeit, der sogenannten Induktionszeit, annähernd linear mit der Zeit anzusteigen. Dies ist immer dann der Fall, wenn sich die Konzentration im unteren Vorratsgefäß während der Meßzeit nur unwesentlich ändert und ihr gegenüber die Konzentration des oberen Vorratsgefäßes vernachlässigbar klein bleibt. Für eine gegebene Geometrie wird dann der Diffusionstransport während der Meßzeit proportional dem Diffusionskoeffizienten und der Ausgangskonzentration $c_{0}$ der Lösung im unteren Vorratsgefäß sein.

$$
J=\frac{\mathrm{d} c}{\mathrm{~d} t} V^{\mathrm{o}} \sim D c_{0}
$$

$\left(V^{0}=\right.$ Volumen des oberen Vorratsgefäßes).
Eine Uberprüfung der Aussage 1, ob der beobachtete Stofftransport auf der Grundlage ungestörter Diffusion erfolgt, kann dann in folgender Weise durchgeführt werden. Bei gleichzeitiger Diffusion der Komponenten eines binären Gemisches ergibt sich für jede der beiden Komponenten eine Proportionalität nach (5), wenn ihre Diffusion weitgehend voneinander unabhängig erfolgt. Nach Eliminierung der Anlaufphase der Diffusion müßte sich also ein Anreicherungsfaktor $\tau$ nachweisen lassen, der dem Verhältnis der Diffusionskoeffizienten beider Komponenten entspricht

$$
\tau=\frac{D_{1}}{D_{2}}=\frac{J_{1}}{J_{2}} \cdot \frac{c_{02}}{c_{01}} .
$$

Eine weitere Probe mußte darin bestehen, diesen Anreicherungsfaktor bei verschiedenen Pulsationsfrequenzen zu ermitteln. Ist ein Durchmischungsanteil am Stofftransport beteiligt, so müßte dieser um so stärker in Erscheinung treten, je rascher die Pulsationsvorgänge erfolgen.

Abb. 5 zeigt die bei der Diffusion eines Lösungsgemisches (Trypaflavin, $c_{0}=0,016$ Gew.-Proz.; Methylenblau, $c_{0}=0,008$ Gew.-Proz.) gegen sein Lö-

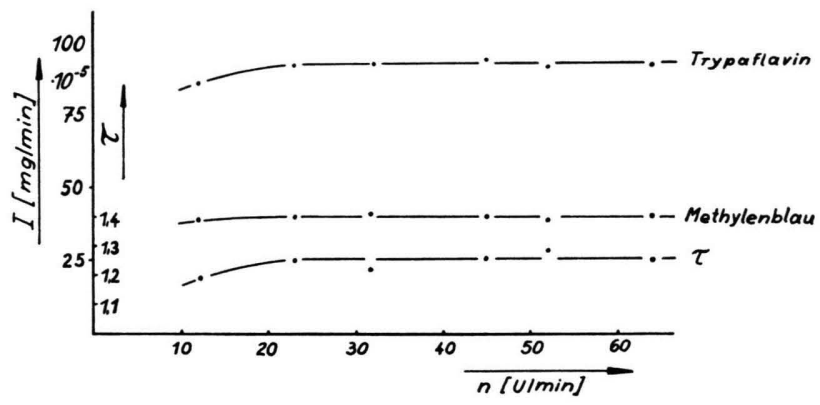

Abb. 5. Abhängigkeit des Diffusionstransportes $J$ für ein Gemisch aus Trypaflavin (0,016 Gew.-Proz.)/Methylenblau (0,008 Gew.-Proz.) in Aqua dest. und des Anreicherungsfaktors $\tau$ von der Pulsationsfrequenz (nach ${ }^{3}$ ).

sungsmittel (Aqua dest.) erhaltenen Werte. Die verwendete Kapillare war $180 \mathrm{~mm}$ lang bei $0,5 \mathrm{~mm} \phi$. Es tritt tatsächlich ein Anreicherungsfaktor von 1,26 auf, der auch etwa dem Diffusionskoeffizientenverhältnis beider Komponenten entspricht (nach NISTLER $^{7}: D_{1} / D_{2}=1,29$ ) und oberhalb einer Exzenterdrehzahl von 20 min $^{-1}$ konstant bleibt.

Die in Abb. 5 mitdargestellten Diffusionsströme für beide Komponenten zeigen in Ủbereinstimmung mit Aussage 2 die Unabhängigkeit des Diffusions-

7 A. Nistler, Kolloidchem. Beih. 28, 296 [1929]. 
transportes von der Pulsationsfrequenz bei genügend häufigem Wechsel der Paraboloide über einen gröBeren Bereich ${ }^{8}$.

Gleichung (4) ist also noch hinsichtlich der Aussagen 3 und $4 \mathrm{zu}$ überprüfen.

Abb. 6 und 7 zeigen die Messungen für Thioharnstoff in Aqua dest. Die maximale Paraboloidhöhe war stets $96 \%$ der Kapillarlänge. Die erhaltenen Resultate sind in Tab. 1 und 2 zusammengestellt.

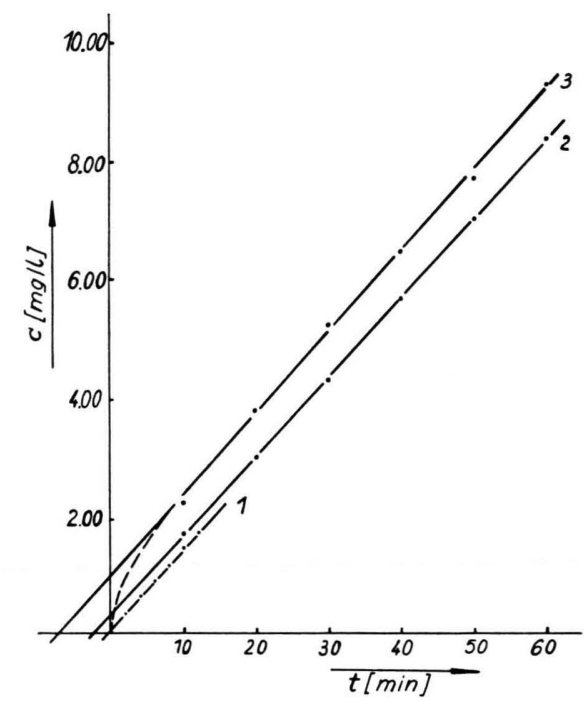

Abb. 6. Zeitlicher Konzentrationsanstieg im oberen Vorratsgefäß für Kapillaren gleicher Länge aber unterschiedlichen Durchmessers: Kurve 1: 0,5 mm; Kurve 2: 1,0 mm; Kurve 3: $2,0 \mathrm{~mm}$. Für Kurve 3 ist die Anlaufphase der Diffusion gestrichelt eingezeichnet; für die anderen Darstellungen nur der lineare Teil und seine Extrapolation auf die Zeitachse (Induktionszeit). Versuchsdaten siehe Tab. 1.

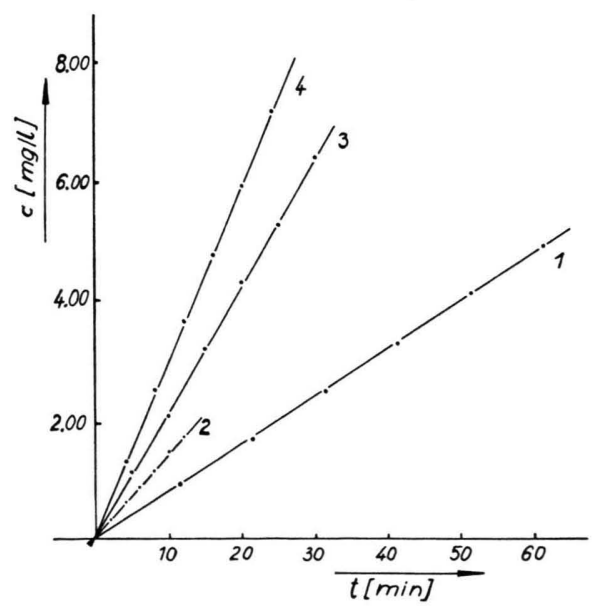

Abb. 7. Zeitlicher Konzentrationsanstieg im oberen Vorratsgefäß für Kapillaren gleichen Durchmessers aber unterschiedlicher Länge: Kurve 1: 100 mm; Kurve 2: $180 \mathrm{~mm}$, Kurve 3: $280 \mathrm{~mm}$; Kurve 4: $380 \mathrm{~mm}$. Versuchsdaten siehe Tab. 2.
In Übereinstimmung mit den zur Abschätzung angestellten Überlegungen ergibt sich für gleich lange Kapillaren trotz unterschiedlichen Durchmessers stets der gleiche Diffusionstransport (Tab. 1). Die Abweichungen vom Mittelwert $J=76,6 \cdot 10^{-6} \mathrm{mg} / \mathrm{sec}$ sind nicht systematisch und nicht größer als $0,5 \%$.

\begin{tabular}{ccccc}
\hline Tabelle & $\begin{array}{c}\text { Kapillare } \\
\text { Länge/ } \varnothing \\
{[\mathrm{mm} / \mathrm{mm}]}\end{array}$ & $\begin{array}{c}\text { Diff.-Transport } \\
J \\
{[\mathrm{mg} / \mathrm{sec}]}\end{array}$ & $\begin{array}{c}\text { Induktionszeit } \\
{[\mathrm{min}]}\end{array}$ & $\begin{array}{c}\text { Verstär- } \\
\text { kungs- } \\
\text { faktor }\end{array}$ \\
\hline \multirow{2}{*}{1} & $180 / 0,5$ & $76,7 \cdot 10^{-6}$ & ca. 0,5 & \\
& $180 / 1$ & $76,2 \cdot 10^{-6}$ & ca. 2,5 & \\
& $180 / 2$ & $76,9 \cdot 10^{-6}$ & ca. 6,0 & \\
2 & $100 / 0,5$ & $46,1 \cdot 10^{-6}$ & ca. 0,5 & 3000 \\
& $180 / 0,5$ & $76,6 \cdot 10^{-6}$ & ca. 0,5 & 5000 \\
& $280 / 0,5$ & $121 \cdot 10^{-6}$ & ca. 0,5 & 8000 \\
& $380 / 0,5$ & $166 \cdot 10^{-6}$ & ca. 0,5 & 11000
\end{tabular}

Tab. 1 und 2. a) Lösung: Thioharnstoff in wäßriger Lösung $c_{0}=300 \mathrm{mg} / \mathrm{l}$. b) Oberes Vorratsgefä $ß: V^{0}=33,3 \mathrm{ml}$.

Die Vergrößerung des Diffusionsweges bei Übergang zu größeren Kapillardurchmessern, ein $\mathrm{Ma}$ dafür ist die Verlängerung der Induktionszeit, wird also in bezug auf den Diffusionstransport durch die vergrößerte Paraboloidoberfläche vollständig kompensiert.

Weiterhin in Übereinstimmung mit (4) ergibt sich ein linearer Zusammenhang zwischen dem Diffusionstransport und der Kapillarlänge (Tab. 2 und Abb. 8). Die Erhöhung des Diffusionstransportes ist dabei nur auf die Vergrößerung der Paraboloidoberfläche zurückzuführen; der Diffusionsweg bleibt etwa konstant (konstante Induktionszeit).

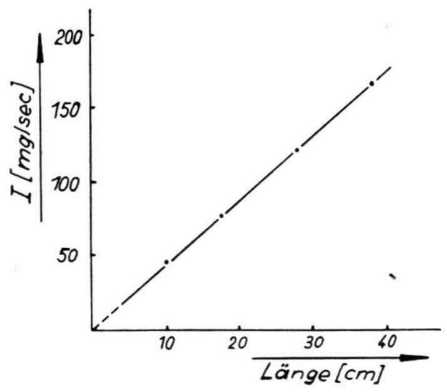

Abb. 8. Diffusionstransport in Abhängigkeit von der Kapillarlänge (nach den Meßwerten der Abb. 7).

8 Bei weiterer Steigerung der Exzenterdrehzahl ist jedoch mit einem plötzlichen Ansteigen des Stofftransportes zu rechnen, wie dies in ${ }^{3}$ bisher für Kapillaren mit größerem Durchmesser nachgewiesen wurde und dessen Klärung noch einer weiteren Bearbeitung bedarf. Diese Erscheinung beginnt weit unter der kritischen Re-Zahl einer stationären Strömung. 
Bevor der Diffusionstransport bei Pulsation mit dem einer konventionellen Anordnung verglichen werden kann, ist erst ein geeigneter Vergleichsmodus zu suchen. Bei einer Pulsationsanordnung wird man bei gegebenem Querschnitt einer Kapillare ihre Länge möglichst groß, bei konventionellen Anordnungen hingegen möglichst klein wählen, da bei letzterer Anordnung der Diffusionsweg durch die Länge der Kapillare gegeben ist. Aus Gründen ungestörter Diffusion wird man jedoch in solcher Anordnung ein Verhältnis von Länge zu Durchmesser der Kapillare von wenigstens $10: 1$ annehmen müssen, d.h. der Diffusionsweg beträgt dann $\Delta x=10 d$.

Im stationären Zustand mit

$$
\Delta c \approx c_{0} ; \quad \Delta x \approx 10 d ; \quad F=\frac{\pi d^{2}}{4}
$$

ergibt sich der Stofftransport einer konventionellen Anordnung aus dem 1. Fickschen Gesetz zu

$$
J_{\text {konv. }}=-D \frac{\Delta c}{\Delta x} F=D c_{0} \frac{\pi d}{40}
$$

Für die in Tab. 1 angegebenen Versuchsbedingungen und ${ }^{9} D=1,3 \cdot 10^{-5} \mathrm{~cm}^{2} / \mathrm{sec}$ wird also der Diffusionstransport

$$
J_{\text {konv. }}=15,2 \cdot 10^{-9} \mathrm{mg} / \mathrm{sec} .
$$

Der Verstärkungsfaktor des Diffusionstransportes einer Pulsationsanordnung zu dem einer konventionellen Anordnung ergibt sich dann aus dem Verhältnis :

$$
\alpha=J_{\text {Pulsation }} / J_{\text {konv. }} \text {. }
$$

In Spalte 4 der Tab. 2 sind die durch Pulsation erhaltenen Verstärkungsfaktoren nach (7) eingetragen.

9 Nach eigenen Messungen.
Verwendet man als Diffusionszone zwischen beiden Vorratsgefäßen einer Anordnung nicht nur eine Kapillare, sondern ein Kapillarbündel aus $n$ Kapillaren, so würde sowohl für eine konventionelle als auch für eine Pulsationsanordnung der gesamte Diffusionstransport $n$-mal größer, d. h. der Verstärkungsfaktor bleibt von der Zahl der Kapillaren unabhängig.

Es ist also möglich, den Diffusionstransport zwischen zwei Vorratsgefäßen bei Pulsationsdiffusion unter praktischen Bedingungen auf das $10^{3}$ - bis $10^{4}$. fache einer konventionellen Anordnung zu steigern. Verbunden mit der im Vergleich zu Gasen höheren Dichte in Flüssigkeiten (außer bei sehr verdünnten Lösungen) kann die Entwicklung eines Diffusionstrennverfahrens nun auch in flüssiger Phase sehr aussichtsreich werden.

Zum Schluß sei noch darauf hingewiesen, daß es nicht zweckmäßig ist, eine Diffusionszone als Pakkung von $n$ Kapillaren auszubilden. Der Durchmesser geht in den Strömungswiderstand einer Kapillare nach dem Hagen-Poiseuilleschen Gesetz mit der 4. Potenz ein, d. h. eine solche Pulsationsanordnung würde außerordentlich hohe Forderungen in bezug auf Gleichmäßigkeit der Durchmesser stellen. Diese Gleichmäßigkeit der Unterteilung einer Diffusionszone ist wesentlich einfacher durch eine Lamellenpackung aus planparallelen Platten, die durch Distanzstreifen aus gleichmäßig gewalztem Material äquidistant gegenüber stehen, zu erreichen.

Für die Messung von Diffusionskoeffizienten erweist sich schon der Diffusionstransport durch eine Kapillare als ausreichend. Über die Bestimmung von Diffusionskoeffizienten mit der Methode der Pulsationsdiffusion und die Erweiterung von (4) auf den quasistationären Zustand wird im noch folgenden Teil II berichtet werden. 